\title{
Repetitive Control and Virtual Bleeder Resistor for AC Generator Sets with Harmonic-Sensitive Loads
}

\author{
L. Fazia*, L. Peretti**, M. Zigliotto** \\ * BFG Engineering srl, Vicenza, Italy, email: postmaster@e-brain.it \\ * Department of Engineering and Management of Industrial Systems, University of Padova, Italy \\ email: luca.peretti@unipd.it, mauro.zigliotto@unipd.it
}

Keywords: AC generator sets, PM synchronous generators, Repetitive Control, Virtual Bleeder Resistor.

\begin{abstract}
Low and medium power full-digital AC generator sets are largely used in both industrial and civil fields. During the last years, more demanding applications for AC generators have emerged. They require more power combined with a cleaner voltage source. Consequently, particular care is requested for both the mechanical and electrical design of a new AC generator set, as well as a keen choice of the digital control strategy. This paper presents a control technique suitable for gasoline engine-driven AC generator sets, which minimises the voltage harmonic distortion by means of repetitive control, while assuring good performances in a very wide load range by means of a virtual bleeder resistor (VBR). Simulation and experimental results confirm the effectiveness of the proposed strategy.
\end{abstract}

\section{Introduction}

Newly developed low and medium power full-digital AC generator sets must cope with the growing and increasingly demanding market of industrial and domestic applications, which require more power combined with a clean voltage source. Examples are represented by home appliances and personal computers, which are considered highly sensitive to the main AC supply harmonic content. Within this frame, the design of a modern gasoline engine-driven AC generator set must look after both the mechanical and electrical aspects [1]. So far, many control strategies have appeared in the literature. The general intuition is that classic PID regulators, in spite of their simplicity, do not cope properly with wide load variations, especially when a very low harmonic distortion in presence of non-linear loads is requested. Most effective solutions exploit the periodical nature of the disturbances, using resonant controllers and repetitive control blocks with different control topologies. In [2], a mixed control strategy for diesel engine-driven AC generators has been proposed. The system uses an inner current control loop with a PID regulator, and an outer voltage control loop with two cascaded resonant controllers, tuned at the voltage fundamental frequency and its third harmonic. In this way, the rejection of the main distortion contribute is achieved. However, steady-state error for the regulated current is expected, due to the sinusoidal nature of both voltage and current references. Moreover, higher voltage harmonic distortions are still present. In [8], a repetitive control block substitutes both voltage and current regulators. This strategy is simple and fits for low-cost applications. However, the problem of possible overcurrents is not considered, and the overall system dynamic relies only on the repetitive controller, which is in general rather slow. Moreover, the problem of wide load variations is not deepened. A viable solution for both wide load variation and harmonic distortion is presented in [6]. A repetitive block is inserted in the outer voltage control loop, and an on-line adaptive scheme based on a recursive least square algorithm estimates the parameters of the voltage closed loop, which is simplified as a second-order transfer function. Repetitive control parameters are adapted according to the estimation, and the matching guarantees an overall stability in the whole range. While computationally more intensive than others, this solution seems effective. Some concerns may arise about the voltage settling time after a step transient, due to the heavy filter action on the estimation output that is needed to cope with non-linear loads. The proposed work deals with the application of a novel control strategy for a $10 \mathrm{kVA}-230 \mathrm{~V}_{\mathrm{RMS}}-50 \mathrm{~Hz}$ gasoline engine-driven $\mathrm{AC}$ generator set, featuring extremely low harmonic distortions even in presence of non-linear loads. The target is reached using a control strategy that recalls those presented in [4] and [5]. As a first improvement, PID regulators in both current and voltage control loops have been substituted by resonant controllers, tuned on the fundamental voltage frequency $(50 \mathrm{~Hz})$. A distinctive feature of the proposed scheme is also the accurate implementation of a virtual bleeder resistor (VBR), which mitigates the effects of wide load variations. There is evidence that the proposed innovations give the system very good performances in terms of output voltage accuracy, low harmonic distortion and dynamic behaviour.

\section{System Description}

In the proposed $\mathrm{AC}$ generator set, an internal combustion engine (ICE) is mechanically coupled to a Permanent Magnet Synchronous Generator (PMSG). The three-phase voltages produced by the PMSG are rectified and filtered, to derive a DC link voltage that feeds a single-phase $10 \mathrm{kVA}$ IGBT inverter prototype. The digital control system has been implemented using a low-cost 32 bit RISC processor board with a PWM frequency of $15 \mathrm{kHz}$, equivalent to a switching 
period $T_{c}=66.67 \mu \mathrm{s}$. Current sampling was synchronised with the PWM, to reduce the aliasing effect due to the voltage (and current) modulation ripple. Output voltage signal sampling was performed at the same rate. A complete scheme of the system is reported in Fig.1.

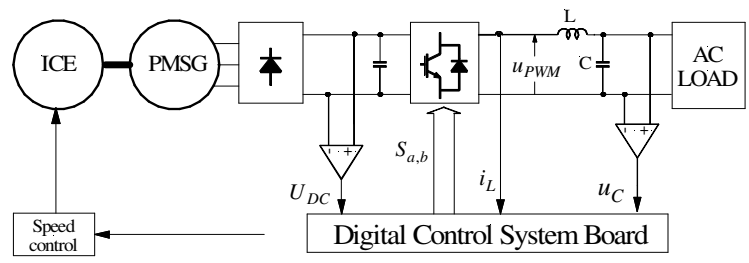

Fig. 1: Block diagram of the Diesel-driven AC power plant.

The PWM output voltage is filtered by a $L C$ circuit before the connection to the AC load, that can be either linear (e.g. resistive heater) or non-linear (e.g. diode rectifier or a phasecontrolled AC motor). The microprocessor-based card acquires the filter inductor current $i_{L}$, the output voltage $u_{C}$ and implements both the inner current loop and an outer voltage loop. As output, the control board delivers the IGBT gate commands $S_{a, b}$, inserting the appropriate dead-times to avoid shoot-through faults. Normally, varying loads cause sensible voltage drops across the PMSG output impedance, and the resulting DC link voltage fluctuations act as a disturbance in the control loop. As a countermeasure, a direct DC link voltage measurement $u_{D C}$ has been realised.

\subsection{Inverter and linear load model}

Let's first suppose that the AC load of Fig. 1 is modelled by a linear resistor $R_{L}$. The transfer function between the PWM output voltage $u_{P W M}$ and the inductor current $i_{L}$ is:

$$
\frac{i_{L}}{u_{P W M}}(s)=\frac{1}{R_{L}} \cdot \frac{s R_{L} C+1}{s^{2} L C+s L / R_{L}+1}
$$

While the transfer function between the inductor current $i_{L}$ and the capacitor voltage $u_{C}$ is:

$$
\frac{u_{C}}{i_{L}}(s)=\frac{R_{L}}{s R_{L} C+1}
$$

Consequently, the transfer function between $u_{P W M}$ and $u_{C}$ is a second-order low-pass filter:

$$
\frac{u_{C}}{u_{P W M}}(s)=\frac{1}{s^{2} L C+s L / R_{L}+1}
$$

With a possible bleeder resistor $R_{B}$ in parallel to the load $R_{L}$, the above transfer functions remain the same, except that $R_{L}$ must be substituted by a new equivalent resistor $R$ :

$$
R=\frac{R_{B} R_{L}}{R_{B}+R_{L}}
$$

$R_{B}$ is a permanently inserted resistor which helps to reduce the range of load variations. Load range

One of the key factors in the control design was the robustness with respect to load variations. Load variations heavily affect the transfer function (1), as depicted in Fig. 2.

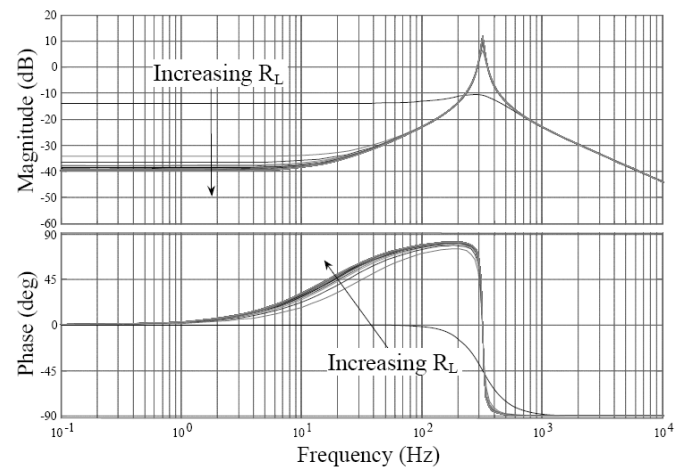

Fig. 2: Transfer function $i_{L}(s) / u_{P W M}(s)$ for different values of $R_{L}$.

Fig. 2 is the base for the current control loop design, and it has been obtained plotting Equation (1) from the no load $\left(R_{L} \rightarrow+\infty\right.$, absence of $\left.R_{B}\right)$ to the full load $\left(10 \mathrm{~kW}, 230 \mathrm{~V}, R_{L}=\right.$ $5.29 \Omega$ ) condition. One of the major difficulties in the control design is to get the same system stability and dynamic behaviour in all of the load conditions. Actually, different working points would require different control parameters to get the same phase margin and bandwidth requirements. As a consequence, unless on-line tuning is a feasible option, the regulator must be tuned with the parameter set that provides the minimum deviation from the specifications in the whole load range. In (1), both the transfer function gain and the damping coefficient of the imaginary poles are inversely proportional to $R_{L}$. The worst condition occurs at no load, when Equation (1) has a gain close to zero and a couple of pure imaginary poles. In this situation, the stability and the dynamic specifications are difficult to achieve. It is easy deducible that if the load range is reduced by a bleeder resistor $R_{B}$ permanently inserted in parallel to $R_{L}$, the transfer function gain and the damping coefficient will never be zero, and the control tuning is easier. In most available $\mathrm{AC}$ generator sets, the choice of $R_{B}$ is the result of a trade-off. On one hand, it enhances the control performances in the whole operating range. On the other, it dissipates a constant power, to the detriment of the system efficiency. The following Section 3 will present a method to create a virtual bleeder resistor, based on firmware only, able to retain the benefits discarding the drawbacks.

\section{The virtual bleeder resistor}

A virtual bleeder resistor can be obtained by some control modifications that can be easily obtained by block algebra. Fig. 3 shows the starting block scheme, that is, the current control loop with the insertion of a physical bleeder resistor.

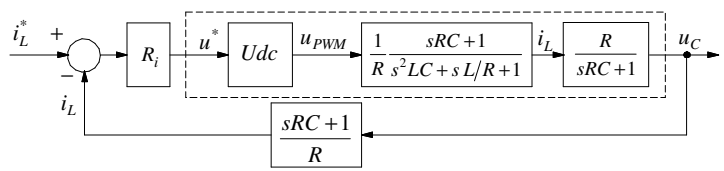

Fig. 3: Current control loop with physical bleeder resistor.

For the sake of the next moves, the inductor current $i_{L}$ feedback signal is obtained from capacitor voltage $u_{C}$, and then processed by the block $(s R C+1) / R$, which is the transfer 
function from $u_{C}$ to $i_{L}$. As a first approximation, the time constant of the PWM voltage generation has been neglected, since it is very small compared to the others. The final scheme will be that of Fig. 4, in which the bleeder resistor is substituted by two transfer functions $F_{l}(s)$ and $F_{2}(s)$.

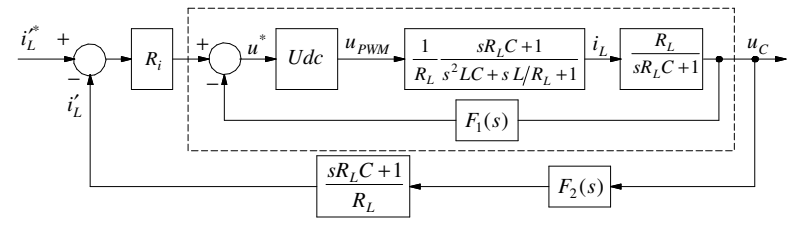

Fig. 4: Current control loop with virtual bleeder resistor.

$F_{l}(s)$ is obtained by forcing the equivalence between the transfer functions within the dashed lines of Fig. 3 and Fig. 4:

$$
\frac{U d c}{s^{2} L C+s L / R_{L}+1+U d c F_{1}(s)}=\frac{U d c}{s^{2} L C+s L / R+1}
$$

From Equation (5), it follows:

$$
F_{1}(s)=\frac{s L}{U_{d c} R_{B}}
$$

Equation (6) is an improper transfer function. Consequently, particular care must be taken in its digital implementation, for example by adding some poles to make it proper. The final expression for $F_{2}(s)$ can be obtained in a similar way, by forcing the same transfer functions for the current control loops in Fig. 3 and Fig. 4:

$$
F_{2}(s)=\frac{R_{B}+R_{L}\left(1+s R_{B} C\right)}{R_{B}+s C R_{B} R_{L}}
$$

It is worth to note that Equation (7) is of little practical value, since it evidently hinges on the load resistance $R_{L}$. Nevertheless, $R_{B}$ plays a crucial rule at no-load operations and a good approximation of Equation (7) can be sought for $R_{L} \rightarrow+\infty$ :

$$
\lim _{R_{L} \rightarrow \infty} F_{2}(s)=\frac{1+s R_{B} C}{s R_{B} C}
$$

The practical implementation of Equation (8) is straightforward, since it has the same structure of a common PI regulator. The final block scheme, with the newly calculated expressions for $F_{1}(s)$ and $F_{2}(s)$, is shown in Fig. 5.

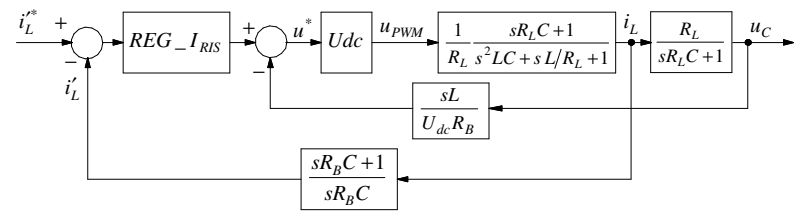

Fig. 5: First implementation of the virtual bleeder resistor.

Last, it is worth to note that the value of $i_{L}{ }_{L}$ in the feedback connection of Fig. 5, is not equal to $i_{L}$. This means that the controller does not control the real sampled current, but a value which is post-processed by $F_{2}(s)$. The original meaning of the current reference signal can be restored introducing a pre-processing block equal to $F_{2}(s)$ just before $i_{L}{ }^{*}$, leading to the final block scheme of Fig. 6.

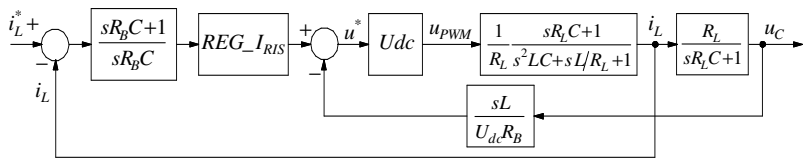

Fig. 6: final implementation of the virtual bleeder resistor.

\section{Resonant current and voltage controllers}

In this work, resonant controllers for the current and voltage control loops have replaced conventional PI regulators. The choice is justified by the fact that voltage amplitude and frequency are fixed $\left(230 \mathrm{~V}_{\mathrm{RMS}}, 50 \mathrm{~Hz}\right)$, and the PMSG is customised to the purpose. Therefore, parameters' ranges are known and resonant controllers can be tuned with relative easiness. They give the system superior accuracy, which is one of the key-specifications in AC generator sets.

\subsection{Current controller}

The transfer function of the resonant current controller is the following:

$$
R E G_{-} I_{R I S}(s)=K_{p i} \frac{\left(1+s \tau_{i i}\right)\left(1+s \alpha_{i}\right)}{s^{2}+\omega_{n}^{2}}
$$

A null steady state error is required at $\omega_{n}=2 \pi(50 \mathrm{~Hz})$, while the zero $\left(1+\mathrm{s} \alpha_{\mathrm{i}}\right)$ guarantees an adequate phase margin in the open-loop transfer function diagram. The current regulator design has a degree of freedom, since three parameters need to be tuned $\left(K_{p i}, \tau_{i i}, \alpha_{i}\right)$ with only two bonds, on phase margin and crossover frequency of the open-loop diagram respectively. It has been experienced that $\alpha_{i}$ tuning is quite relaxed, and it can be optimised by simulation for smart performances in the whole load range. The Bode diagrams of the open loop transfer function at no-load and at full load are reported in Fig. 7.

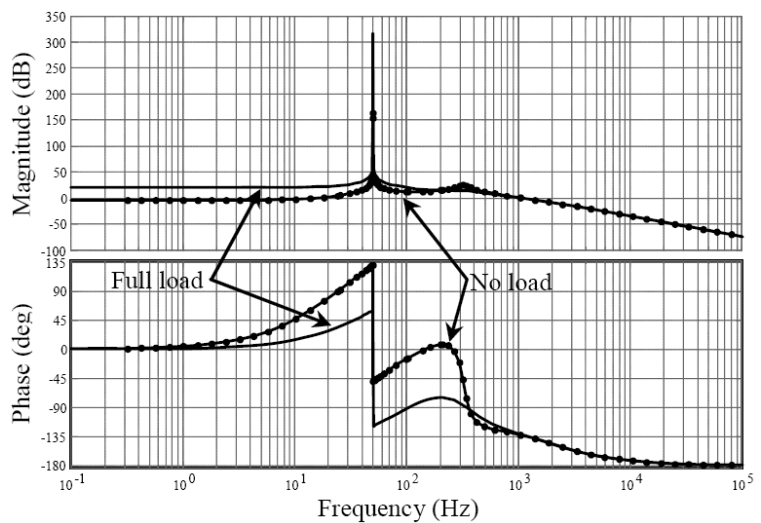

Fig. 7: Open loop Bode diagram for the current control loop.

The phase margin is approximately $0.8 \mathrm{rad}$ in the whole load range. However, the major advantage of the resonant controller emerges in the closed-loop Bode diagram. Opposite to a common PI regulator, which shows a gain less than unity 
and a finite phase-lag at $50 \mathrm{~Hz}$, the resonant controller presents a unity gain and a zero phase-lag at the same frequency for all operating conditions. Obviously, this comes from the very high gain at $50 \mathrm{~Hz}$ in the open loop Bode diagram, as shown in Fig. 7.

\subsection{Voltage controller}

The resonant voltage controller has the same structure of the current controller in Equation (9). As a consequence, the voltage controller design is like that of the current controller, with similar open-loop Bode diagram. A crossover frequency of $300 \mathrm{~Hz}$ has been imposed, distant from that of the current control loop, to avoid the interference between inner and outer loops. The phase margin is approximately $1.3 \mathrm{rad}$ for all the operating conditions.

\section{Repetitive control}

In the previous sections, the load has been always considered a linear resistor. Most likely, non linear loads are connected to the generator, for example diode rectifiers. In this case, the resonant (or PID) controllers action, combined with the inverter PWM and the $L C$ filter, might not be sufficient for an effective reduction of the harmonics. The output voltage total harmonic distortion (THD) can be highly improved by a repetitive controller. Repetitive controllers are particularly suitable for a digital implementation. They rely on the Internal Model Principle (IMP) theory [7], which states that the tracking/rejection of periodical references/disturbances can be obtained if the regulator incorporates the transfer function of the signal to be tracked/rejected. In the present case, with a reference signal at $50 \mathrm{~Hz}$ and periodical disturbances at frequencies multiple of the fundamental one, the voltage controller is modified as shown in Fig. 8, where a repetitive block is inserted in parallel to the resonant controller.

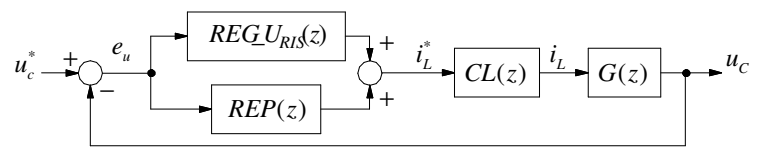

Fig. 8: Block scheme of the modified voltage control loop.

$C L(z)$ is an approximation of the current control loop transfer function, while $G(z)$ is the transfer function between the inductor current and the capacitor voltage. $R E G_{-} U_{R I S}(z)$ is the resonant voltage regulator discussed in the previous section, and it is the responsible of the dynamics during fast transients. Besides, the repetitive block $R E P(z)$ implements an accurate reference tracking and disturbance rejection during steady state operations. The different behaviour of the two voltage regulators is made possible by a proper gain tuning. From a mathematical point of view, $R E P(z)$ has the following Laplace domain expression [7]:

$$
R E P(s)=\frac{1}{1-e^{-T_{p} s}}
$$

Equation (10) has an infinite gain at the fundamental harmonic frequency $f_{1}=1 / T_{p}(50 \mathrm{~Hz}$ in our case $)$, and at all its integer multiples. The digital (Zeta-transform) domain representation of the repetitive block is easily obtained substituting $z=e^{j \omega T_{c}}$. By imposing that $f_{l}$ is an exact multiple of the sample time $\left(T_{p}=M T_{c}\right)$, it is then

$$
R E P(z)=\frac{1}{1-z^{-M}}=\frac{z^{M}}{z^{M}-1}
$$

Equation (11) represents a positive-feedback loop with a delay of $M$ samples between input and output. $M$ represents the ratio between the sample frequency $f_{c}$ and $f_{l}$ :

$$
M=\frac{f c}{f_{1}}=\frac{15000}{50}=300
$$

The repetitive controller is implemented in the digital domain with the scheme depicted in Fig. 9, which highlights two modifications with respect to Equation (11).

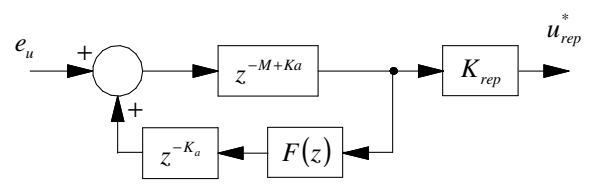

Fig. 9: Digital implementation of the repetitive control block.

The first one is the introduction of a lag of $M-K_{a}$ samples instead of $M$ samples in the direct connection, compensated by a $K_{a}$ lag in the feedback path. In this way, the poles map is the same, but the phase margin is enhanced and $K_{a}$ leading action can be used to compensate the delay due to the PWM. The second implementation hint is the introduction of a FIR filter $F(z)$ in the feedback connection, which smoothes the repetitive amplitude peaks at high frequencies, avoiding possible oscillations caused by the noise. The repetitive dynamic is tuned by the gain $K_{\text {rep }}$. Its action has to be harmonised with that of the resonant controller action to get a precise and fast control in all operating conditions. As a remark, the name "repetitive" originates from the nature of the scheme in Fig. 9, in which a sample is redirected to the input by the feedback loop after $M$ control cycles.

\section{Simulation and experimental results}

The AC generator set described in the paper has been implemented on a pre-industrialised prototype, for a series of tests, before its introduction to the market. The control board is based on the Renesas SH7125 low-cost microprocessor, with RISC architecture and $50 \mathrm{MHz}$ clock frequency. The board uses the internal 10 bit A/D converters and PWM generator. The whole algorithm (including VBD, resonant controllers and repetitive add-on feature) is executed every $66.6 \mu \mathrm{s}(15 \mathrm{kHz})$, with a CPU load factor of about $50 \%$. The remaining time is used for general purpose $\mathrm{I} / \mathrm{O}$ management, including the command to the ICE throttle. The software has been developed in $\mathrm{C}$ language, which is the right compromise between development and execution times. 
Fig. 10 shows the transients of output voltage and current, during abrupt loading from no-load to $50 \%$ of the full load (linear). Symmetrically, Errore. L'origine riferimento non è stata trovata. reports the same quantities at load detach.

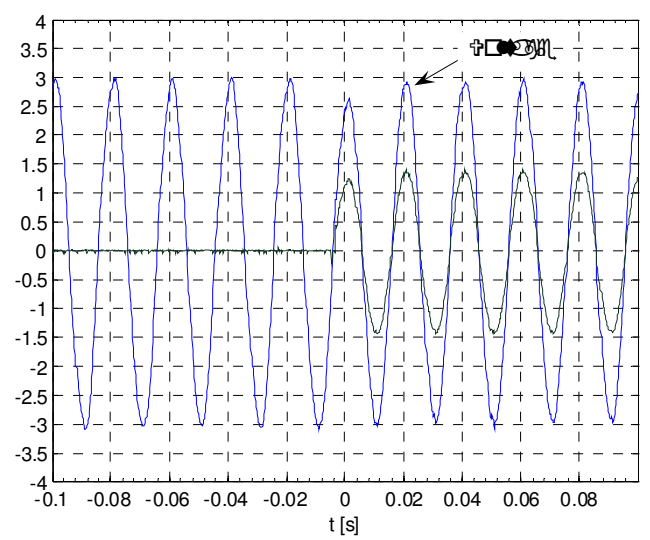

Fig. 10: Experimental output voltage transient at loading (y-scale: voltage $1 \mathrm{~V}=100 \mathrm{~V}$, current $1 \mathrm{~V}=20 \mathrm{~A}$ ).

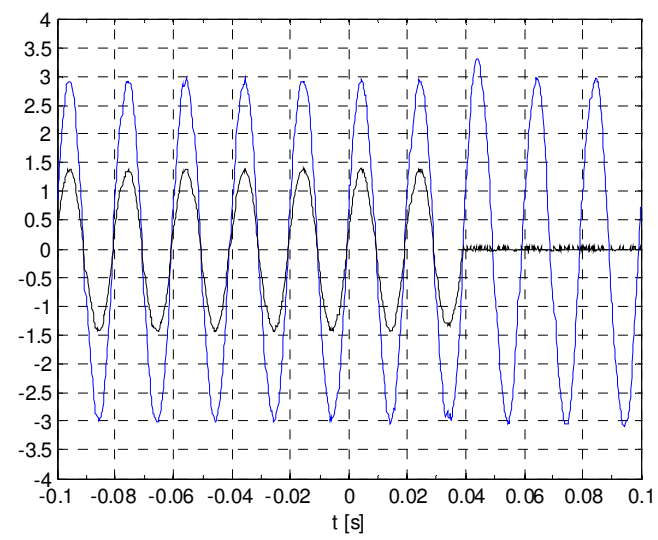

Fig. 11: Experimental output voltage transient at load detach (y-scale: voltage $1 \mathrm{~V}=100 \mathrm{~V}$, current $1 \mathrm{~V}=20 \mathrm{~A}$ ).

The experimental figures show that the voltage output is restored within one cycle of the sinusoidal output, and the amplitude variation is around $\pm 11 \%$, within the most stringent requirements of the related norm (The International Standard ISO 8528-3 "Alternating current generators for generating sets", performance class G3). Many other working conditions have been preliminary tested by simulation, which is based on an accurate model of the whole system, including PWM dead times and LC filter parasitic components. They constitute the schedule for the next laboratory measurement activity.

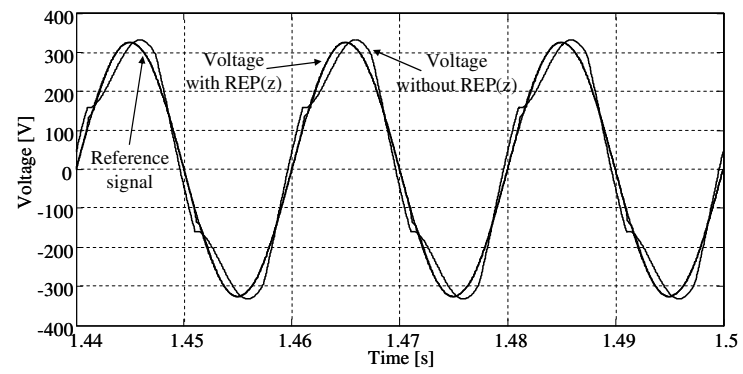

Fig. 11: Output voltage with and without repetitive and non linear load.
For example, Fig. 11 shows the output voltage in case of non linear load, constituted by a diode rectifier cascaded with an $R C$ parallel $(\mathrm{R}=12 \Omega, \mathrm{C}=500 \mu \mathrm{F})$. As it can be seen, without the repetitive block the voltage distortion is quite high ( $\mathrm{THD}=14.9 \%$ ), while the presence of the repetitive restores the sinusoidal profile (THD=1.5\%).

\section{Conclusions}

The paper presents a comprehensive study, design and development of a performing AC generator set. Some innovations, as virtual bleeder output resistor (for augmented efficiency) and repetitive-based voltage harmonic abatement make the system quite competitive also from environmental point of view.

\section{Acknowledgements}

The financial support of ITALSEA srl (Italy) is gratefully acknowledged. Authors also thanks ing. M. Spagnolo for the kind technical contribution that has made this work possible.

\section{References}

[1] K. Atallah, J. Wang, D. Howe, "Torque-Ripple Minimization in Modular Permanent-Magnet Brushless Machines", IEEE Trans. on Ind. Appl., Vol. 39, No. 6, pp. 1689-1695, (2003).

[2] S. Bolognani, A. Venturato, M. Zigliotto, "Novel Control Technique for High-Performance Diesel-Driven AC Generator-Sets", Proc. of IEE Int. Conf. on Power Electronics and Variable Speed Drives, PEVD 2000, pp. 518-523, London, UK, (2000).

[3] K-S. Kim, B-K. Kwon, C-H. Choi, "A Novel Control Algorithm of a Three-phase PWM Inverter with Output LC Filter", Proc. of IEEE Int. Electric Machines \& Drives Conf., Vol.1, pp.77-81, IEMDC '07, (2007).

[4] P. Mattavelli, L. Tubiana, M. Zigliotto, "Torque Ripple Reduction in PM Synchronous Motor Drives Using Repetitive Current Control", IEEE Trans. on Pow. Electr., Vol. 20, No. 6, pp. 1423-1431, (2005).

[5] C. Rech, H. Pinheiro, H. A. Grundling, H. L. Hey, J. R. Pinheiro, "Analysis and Design of a Repetitive Predictive-PID Controller for PWM Inverters", IEEE 32nd Annual Power Electronics Specialists Conference, PESC'01, Vol.2, pp.986-991, (2001).

[6] Y-Y. Tzou, S-L. Jung, H-C. Yeh, "Adaptive Repetitive Control of PWM Inverters for Very Low THD AC Voltage Regulation with Unknown Loads", IEEE Trans. on Pow. Electr., Vol. 14, No. 5, pp. 973-981, (1999).

[7] Y. Yamamoto, "Learning Control and Related Problems in Infinite-Dimensional Systems," in Proc. 1993 Eur. Control Conf. , pp. 191-222, (1993).

[8] K. Zhang, Y. Kang, J. Xiong, J. Chen, "Direct Repetitive Control of SPWM Inverter for UPS Purpose", IEEE Trans. on Pow. Electr., Vol. 18, No. 3, pp. 784-792, (2003). 